\title{
Dual-Modified Liposome for Targeted and Enhanced Gene Delivery into Mice Brain ${ }^{\mathbf{s}}$
}

\author{
Bruna dos Santos Rodrigues, Sushant Lakkadwala, Takahisa Kanekiyo, and Jagdish Singh \\ Department of Pharmaceutical Sciences, School of Pharmacy, College of Health Professions, North Dakota State University, \\ Fargo, North Dakota (B.S.R., S.L., J.S.) and Department of Neuroscience, Mayo Clinic, Jacksonville, Florida (T.K.)
}

Received November 22, 2019; accepted June 9, 2020

\begin{abstract}
The development of neuropharmaceutical gene delivery systems requires strategies to obtain efficient and effective brain targeting as well as blood-brain barrier (BBB) permeability. A braintargeted gene delivery system based on a transferrin (Tf) and cell-penetrating peptide (CPP) dual-functionalized liposome, CPP-Tf-liposome, was designed and investigated for crossing BBB and permeating into the brain. We selected three sequences of CPPs [melittin, Kaposi fibroblast growth factor (KFGF), and penetration accelerating sequence-R8] and compared their ability to internalize into the cells and, subsequently, improve the transfection efficiency. Study of intracellular uptake indicated that liposomal penetration into bEnd. 3 cells, primary astrocytes, and primary neurons occurred through multiple endocytosis pathways and surface modification with Tf and CPP enhanced the transfection efficiency of the nanoparticles. A coculture in vitro BBB model reproducing the in vivo anatomophysiological complexity of the biologic barrier was developed to characterize the penetrating properties of these designed liposomes. The dual-functionalized liposomes effectively crossed the in vitro
\end{abstract}

\section{Introduction}

The potential of gene therapy to restore normal cellular functions has raised interest in treatment of devastating diseases such as neurodegenerative diseases (Costantini et al., 2000). Great efforts have been directed toward the development of gene vectors that are able to deliver therapeutic nucleic acids intracellularly in a specific and targeted manner. A wide range of different types of vectors, such as inorganic particles and peptide-based, lipid-based, or polymer-based vectors, has been studied; however, their unsatisfactory gene transfer efficacy has limited their progression as brain-targeted gene delivery carriers (Cook et al., 2015;

This work was supported by National Institutes of Health [Grant R01AG051574]. B.S.R. was supported by doctoral fellowship from the Brazilian National Council for Scientific and Technological Development [221327/20142].

https://doi.org/10.1124/jpet.119.264127.

S This article has supplemental material available at jpet.aspetjournals.org. barrier model followed by transfecting primary neurons. Liposome tissue distribution in vivo indicated superior ability of kFGF-Tf-liposomes to overcome BBB and reach brain of the mice after single intravenous administration. These findings demonstrate the feasibility of using strategically designed liposomes by combining Tf receptor targeting with enhanced cell penetration as a potential brain gene delivery vector.

\section{SIGNIFICANCE STATEMENT}

Rational synthesis of efficient brain-targeted gene carrier included modification of liposomes with a target-specific ligand, transferrin, and with cell-penetrating peptide to enhance cellular internalization. Our study used an in vitro triple coculture bloodbrain barrier (BBB) model as a tool to characterize the permeability across BBB and functionality of designed liposomes prior to in vivo biodistribution studies. Our study demonstrated that rational design and characterization of BBB permeability are efficient strategies for development of brain-targeted gene carriers.

ABBREVIATIONS: $\beta$-gal, $\beta$-galactosidase; BBB, blood-brain barrier; CPP, cell-penetrating peptide; DMEM, Dulbecco's modified Eagle's medium; DOPE, 1,2-dioleoyl-sn-glycero-3-phosphoethanolamine; DOTAP, 1,2-dioleoyl-3-trimethylammonium-propane; kFGF, Kaposi fibroblast growth factor; lip, liposome; Mel, melittin; MTT, 3-(4,5-dimethylthiazol-2-yl)-2,5-diphenyltetrazolium bromide; Na-F, sodium-fluorescein; Pas, penetration accelerating sequence; pDNA, plasmid DNA; PEG, poly (ethylene glycol); pGFP, plasmid GFP; TEER, transendothelial electrical resistance; TEM, transmission electron microscopy; Tf, transferrin. 
increased interbilayer repulsion provided by PEG chains on liposome surface avoids nanoparticle aggregation, consequently improving liposome stability (Immordino et al., 2006).

Cell-penetrating peptides $(\mathrm{CPPs})$ are functional carrier peptides of less than 30 amino acids that infiltrate live cells, in a noninvasive manner, and facilitate the cellular uptake of a variety of bioactive molecules ((de Figueiredo et al., 2014)De Figueiredo et al., 2014; Ramsey and Flynn, 2015). Several CPPs have been successfully used for the delivery of exogenous molecules, including oligonucleotides and proteins, and conjugation of these peptides to liposomes has demonstrated ability to enhance liposome-mediated transfection in cell cultures and in vivo (Khalil et al., 2006, 2007; Zhang et al., 2006; Kim et al., 2010). In this study, we compared the potential of three different CPPs to enhance the gene delivery properties of CPP-coupled liposomes. Melittin (Mel) is a 26-amino-acid-long peptide derived from bee venom and presents strong interaction with plasma membrane promoting its rearrangement with subsequent formation of transmembrane pores, which facilitate the transport of cargo into the cell (Lundquist et al., 2008; Qian and Heller, 2015; Pino-Angeles and Lazaridis, 2018). Mel derivatives have shown to enhance intracellular delivery of therapeutic macromolecules (Kyung et al., 2018). Hydrophobic sequences such as Kaposi fibroblast growth factor (kFGF) have been reported relevant for intracellular delivery of nucleic acids, providing stable noncovalent DNA complexation and protection against nucleases together with the ability to translocate across the lipid bilayer of plasma membrane (Lin et al., 1995; Upadhya and Sangave, 2016; Bolhassani et al., 2017). The addition of hydrophobic sequence FFLIPKG, known as penetration accelerating sequence (Pas), to the arginine-rich peptide R8 formed the hybrid PasR8, revealed not only to improve the carrier abilities but also to facilitate the escape from endocytic lysosomes (Takayama et al., 2009, 2012).

Similar to most of pharmaceutical products, CPPs also face limitations, which include short duration of action, entrapment in endocytic vesicles, and poor tissue specificity (Kristensen et al., 2016). Specific cellular targeting for delivery systems can be addressed by conjugating selective moieties on the surface of the nanoparticles (Zylberberg et al., 2017). Studies focused on braintargeted delivery have extensively explored transferrin (Tf) receptor as a potential transporting route through blood-brain barrier (BBB) (Johnsen and Moos, 2016). Here, we developed plasmid DNA (pDNA)-loaded liposomes bearing CPP and Tf ligands. We explored the properties of liposomal nanoparticulate system for targeted and enhanced gene delivery into brain parenchyma by comparing the abilities of three different types of CPPs (Mel, kFGF, and PasR8) to improve transferrin receptor-targeted liposome delivery properties. In addition, we investigated the transport processes and mechanisms by which CPP-Tf-liposomes cross the in vitro BBB model and, subsequently, transfect primary neurons. Thus, this evaluation will provide an initial inference of in vivo efficacy of nanoparticles to translocate across the BBB as well as allow the optimization of the development of brain-targeted gene carriers.

\section{Material and Methods}

Synthesis of CPP- and Tf-Coupled DSPE-PEG. CPP [Mel (GIGAVLKVLTTGLPALISWIKRKRQQ), kFGF (AAVALLPAVLLALLAP), and PasR8 (FFLIPKGRRRRRRRRGC); Ontores Biotechnologies, Hangzhou, China] and holo-Tf (Sigma-Aldrich, St. Louis,
MO) were conjugated with terminal Succinimidyl ester (NHS)-activated DSPE-PEG ${ }_{2000}$ (Biochempeg Scientific Inc., Watertown, MA) through nucleophilic substitution reaction, separately. Briefly, CPP and DSPE-PEG ${ }_{2000}$-NHS were mixed in anhydrous Dimethylformamide (DMF) at 1:5 molar ratio and adjusted $\mathrm{pH}$ to $8.0-9.0$ with triethylamine (Cai et al., 2014; Layek et al., 2014). After 120 hours under moderate stirring at room temperature, the resulting reaction product was dialyzed (dialysis membrane with molecular weight cutoff of $3500 \mathrm{Da}$ ) for 48 hours.

Tf and DSPE-PEG $2000^{-N H S}\left(125 \mu \mathrm{g}\right.$ of $\mathrm{Tf} / \mu \mathrm{M}$ of DSPE-PEG $\mathrm{D}_{2000^{-}}$ NHS) were mixed in anhydrous Dimethylformamide (DMF) and adjusted $\mathrm{pH}$ to $8.0-9.0$ with triethylamine (Li et al., 2009; Sharma et al., 2014; (Dos Santos Rodrigues et al., 2018)dos Santos Rodrigues et al., 2019a). After 12 hours of reaction, the mixture was dialyzed (dialysis membrane MWCO of $3500 \mathrm{Da}$ ) for 48 hours.

Preparation of Liposomal Formulations. CPP-PEG-DSPE (4 mol\%) along with 1,2-dioleoyl-sn-glycero-3-phosphoethanolamine (DOPE)-1,2-dioleoyl-3-trimethylammonium-propane (DOTAP)cholesterol (45:45:2 mol\%, respectively) were used to prepare CPPliposomes using thin lipid hydration method (Layek et al., 2014; Sharma et al., 2014; Liu et al., 2017). PBS (pH 7.4) was used to hydrate the thin lipid film. Tf-micelles ( $4 \mathrm{~mol} \%$ ) were added to CPP-liposomes formed in the previous process and mixed overnight resulting in TfCPP-liposomes. Plasmid DNA was complexed to chitosan at molar ratios of the amine groups in chitosan and phosphate groups in DNA (N/P) of five and then inserted into the liposomal formulations. Fluorescent-labeled liposomes were prepared by incorporating 1,2dioleoyl-sn-glycero-3-phosphoethanolamine-N-(lissaminerhodamine B sulfonyl) (Avanti Polar Lipids, Alabaster, AL) at $0.5 \mathrm{~mol} \%$ to liposomes. The particle size and zeta potential of liposomal formulations were determined via dynamic light scattering using Zetasizer Nano ZS 90 (Malvern Instruments, Malvern, UK) at $25^{\circ} \mathrm{C}$. Morphology of liposomal formulation was observed by transmission electron microscopy (TEM; JEM-2100, JEOL Ltd., Tokyo, Japan). Hoechst $33342(0.15 \mu \mathrm{g} / \mathrm{ml})$ was used to determine the percent of encapsulated chitosan-pDNA complexes into liposomes, and fluorescence intensity was measured using SpectraMax spectrophotometer (Molecular Devices, San Jose, CA) at $\lambda_{\mathrm{ex}} 354$ and $\lambda_{\mathrm{em}} 458 \mathrm{~nm}$. Percent encapsulation was calculated as fluorescence signal Hoechst 33342 divided by the fluorescence following addition of 0.5\% Triton X-100 (Layek et al., 2014; Liu et al., 2017).

Protection Assay Against Nuclease Degradation. Liposomal formulations (Mel-lip, Mel-Tf-lip, kFGF-lip, kFGF-Tf-lip, PasR8-lip, and PasR8-Tf-lip) containing $1 \mu \mathrm{g}$ pDNA were incubated in the presence of DNase I ( 1 unit) for 1 hour at $37^{\circ} \mathrm{C}$ (Wang et al., 2011). Then, $5 \mu$ l of EDTA $(100 \mathrm{mM})$ was added to terminate the enzymatic reaction. Next, $20 \mu \mathrm{l}$ of heparin $(5 \mathrm{mg} / \mathrm{ml})$ was added to the samples and incubated for 2 hours at room temperature. The released pDNA samples were loaded on $0.8 \%(\mathrm{w} / \mathrm{v})$ agarose gel stained with Ethidium bromide (EtBr) $(0.5 \mu \mathrm{g} / \mathrm{ml})$ and electrophoresed at $80 \mathrm{~V}$ in $0.5 \mathrm{X}$ Trisacetate-EDTA (Bio-Rad, Hercules, CA) buffer for 80 minutes. Free pDNA in the absence or presence of DNase I served as negative control and positive control, respectively.

Cell Culture and Animals. All cell types were cultured at $37^{\circ} \mathrm{C}$ in humidified cell culture incubator and $5 \% \mathrm{CO}_{2}$. The cell line bEnd.3 obtained from American Type Culture Collection (Manassas, VA) was cultured in Dulbecco's modified Eagle's medium (DMEM) growth medium containing $10 \% \mathrm{v} / \mathrm{v}$ FBS and $1 \% \mathrm{v} / \mathrm{v}$ antibiotics. Primary astrocytes and primary neurons were obtained from brain of 1-day-old rats (Sumners and Fregly, 1989). After meninges and vessels were carefully removed, the brains were mechanically and enzymatically [DMEM containing $0.25 \%$ trypsin and DNase I ( $8 \mu \mathrm{g} / \mathrm{ml})$ ] dissociated. Astrocytes obtained from the dissociated cells of brain were maintained in DMEM supplemented with $10 \% \mathrm{v} / \mathrm{v}$ FBS and $1 \% \mathrm{v} / \mathrm{v}$ antibiotics. Purity of astrocyte cultures was checked by immunostaining for glial fibrillary acidic protein antibody. Primary neurons were obtained by treating the dissociated cells with $10 \mu \mathrm{M}$ cytosine arabinoside at day 3. Medium was replaced after 48 hours, and cells 
were allowed to grow for 10 more days. Primary neurons were cultured in DMEM containing $10 \% \mathrm{v} / \mathrm{v}$ plasma-derived horse serum and $1 \% \mathrm{v} / \mathrm{v}$ antibiotics. Purity of neurons was checked by immunostaining for anti-MAP2 antibody.

All the procedures and handling of rats and mice were approved by the Institutional Animal Care and Use Committee at North Dakota State University. Male and female Sprague-Dawley rats (Charles River Laboratories, Wilmington, MA) and male and female C57BL/6 mice (Jackson Laboratories, Bar Harbor, ME) were housed under standard conditions with free access to food and water.

Cytotoxicity Assay. Cytotoxicity of Mel-lip, Mel-Tf-lip, kFGF-lip, kFGF-Tf-lip, PasR8-lip, and PasR8-Tf-lip in bEnd.3 cells, primary astrocytes, and primary neurons was assessed by 3-(4,5-dimethylthiazol-2-yl)-2,5-diphenyltetrazolium bromide (MTT) assay (Sharma et al., 2012). The cells were seeded on 96 -well plates $\left(1 \times 10^{4}\right.$ cells/ well) 24 hours before the experiment. Cells were exposed to liposomal formulations at different phospholipid concentrations (100, 200, 400, and $600 \mathrm{nM}$ ) for 4 hours. Subsequently, cells were washed twice with PBS and cultured with fresh medium for another 48 hours. Then, $10 \mu \mathrm{l}$ of MTT $(5 \mathrm{mg} / \mathrm{ml}$ ) was added in each well. After 3 hours of incubation at $37^{\circ} \mathrm{C}$, the resulting crystals were dissolved by DMSO $(100 \mu \mathrm{l})$, and absorbance was measured at $570 \mathrm{~nm}$. Cells not exposed to liposomal formulations were used as control, and cell viability was calculated and expressed as the percentage of the control.

Cellular Internalization Study. Fluorescent-labeled liposomes were used to investigate the internalization of liposomal nanoparticles into the cells. The cells (bEnd. 3 cells, primary astrocytes, and primary neurons) were cultured on 24-well plates 24 hours before the experiment. Lissamine rhodamine-labeled liposomes $(100 \mathrm{nM})$ were incubated for predetermined times $(0.1,0.25,0.5,1$, and 4 hours). After incubation, suspended formulations were removed, and the cells were washed three times with PBS. Cell membrane was lysed with $0.5 \% \mathrm{v} / \mathrm{v}$ Triton X-100, and lissamine rhodamine was extracted in methanol. Fluorescence intensity was measured by fluorescence spectrometry at $\lambda_{\text {ex }} 553$ and $\lambda_{\text {em }} 570 \mathrm{~nm}$.

Internalization Mechanisms. For investigation of mechanisms of internalization of liposomal formulations, the aforementioned cells were exposed to endocytosis inhibitors: sodium azide $(10 \mathrm{mM})$, chlorpromazine $(10 \mu \mathrm{g} / \mathrm{ml})$, amiloride $(100 \mu \mathrm{g} / \mathrm{ml})$, and colchicine $(50 \mu \mathrm{g} / \mathrm{ml})$ for 30 minutes (dos Santos Rodrigues, 2019a). Then, fluorescent-labeled liposomes (100 nM) were incubated for 4 hours. Subsequently, medium was removed, and cells were washed three times with PBS. Cell membranes were lysed with $0.5 \% \mathrm{v} / \mathrm{v}$ Triton X-100 and lissamine rhodamine extracted in methanol. Fluorescence intensity was measured by spectrofluorometric method ( $\lambda_{\text {ex }} 553$ and $\lambda_{\text {em }} 570 \mathrm{~nm}$ ).

In Vitro Transfection Efficiency. Liposomal formulations (100 $\mathrm{nM}$ ) encapsulating $1 \mu \mathrm{g}$ of chitosan-pDNA complexes were added to bEnd.3 cells, primary astrocytes, and primary neurons and incubated for 4 hours (Sharma et al., 2012). Subsequently, medium was removed; cells were washed three times with PBS and incubated in fresh medium for 48 hours. Percent of cells expressing GFP was evaluated using flow cytometer (FACS analysis; BD Accuri C6, BD Biosciences, San Jose, CA). Quantification of $\beta$-galactosidase $(\beta$-gal) activity was performed using $\beta$-gal assay kit. Total protein levels were determined by BCA assay.

Blood Compatibility Study. The test was performed following the method previously described (dos Santos Rodrigues et al., 2018). Briefly, blood was withdrawn from Sprague-Dawley rats and washed three times with PBS ( $\mathrm{pH} 7.4$ ). Then, $500 \mu \mathrm{l}$ of either different concentrations of liposomal formulations (31.25-1000 nM) or only PBS (negative control) or 1\% Triton X-100 (positive control) was incubated with $1.5 \times 10^{7}$ erythrocytes $(500 \mu \mathrm{l})$ for 1 hour at $37^{\circ} \mathrm{C}$. After incubation, the samples were centrifuged ( $1500 \mathrm{rpm}$ for 10 minutes), and released hemoglobin in the supernatant was measured as absorbance at $540 \mathrm{~nm}$. Hemolysis percentage was calculated considering the absorbance in presence of Triton X-100 as 100\%.

Transport of Liposomes across the In Vitro BBB Model. The designed in vitro BBB model constituted of primary astrocytes $(1.5 \times$ $10^{4}$ cells $/ \mathrm{cm}^{2}$ ) cultured on the lower surface of membrane inserts and
bEnd.3 cells $\left(1.5 \times 10^{4}\right.$ cells $\left./ \mathrm{cm}^{2}\right)$ cultured on upper surface of membrane inserts (Nakagawa et al., 2009; Helms et al., 2016). Transendothelial electrical resistance (TEER) was monitored using EVOM $^{2}$ (World Precision Instruments, Sarasota, FL). After the development of tight contact among cells, fluorescent-labeled liposomes (100 nM) were added to the upper compartment, and fluorescence intensity of liposomes in the basolateral compartment was quantified over a period of 8 hours. TEER was measured before and after transport study to verify membrane integrity. Sodiumfluorescein (Na-F, MW: $376.275 \mathrm{~g} / \mathrm{mol})(100 \mu \mathrm{g} / \mathrm{ml})$, a barrier integrity marker, was used to determine the permeability of the in vitro model and compared with the permeability of liposomal formulations.

Transfection Efficiency of Liposomes in the Triple Cell Culture Model. Liposomal formulations encapsulating $1 \mu \mathrm{g}$ chitosan-pDNA complexes were added to the upper compartment of inserts placed in plates with primary neurons cultured on the bottom. Inserts were removed and media were replaced by fresh media after 8 hours, and cells were incubated for 48 hours. Percent of cells expressing GFP was evaluated by flow cytometry and fluorescence microscopy.

In Vivo Biodistribution and Biocompatibility. Fluorescentlabeled liposomal formulations were administrated via tail vein of C57BL $/ 6$ mice at a dose of $\sim 15.2 \mu \mathrm{mol} / \mathrm{kg}$ body weight $(n=6)$ (Sharma et al., 2013). Fluorescence intensity of lissamine rhodamine was analyzed after 24 hours in the mice and individual organs using neutral infrared imaging. Tissues were weighted and homogenized with PBS, and the dye extracted with chloroform:methanol (2:1). Fluorescence intensity was measured using spectrophotometer at $\lambda_{\text {ex }}$ 560 and $\lambda_{\text {em }} 580 \mathrm{~nm}$. Data were normalized with the negative control treated with saline only. Tissue characteristics and the possible morphologic changes after treatment were analyzed in tissue sections with H\&E staining.

Statistical Analysis. The in vitro studies were performed in four independent experiments, and all data were expressed as means \pm S.D. Statistical significance were evaluated using one-way analysis of variance followed by Tukey's multiple comparison post hoc test using the software GraphPad Prism version 5.0. $P$ values $<0.05$ were considered statistically significant. Six mice per in vivo experimental condition were used to provide statistically valid data.

\section{Results}

Characterization of Liposomal Formulations. Liposomal formulations were prepared according to our previously reported method (dos Santos Rodrigues et al., 2018), and they were optimized to generate highly multivalent nanoparticles that could cross the BBB efficiently. We modified the surface of liposomes with Tf ligand for targeting the transferrin receptors, which are expressed on the surface of brain capillary endothelial cells, and a CPP to enhance cell penetration. The lipid vesicles were obtained with a mean size of $153 \mathrm{~nm}$. Incorporation of $\mathrm{Tf}$ on liposome surface led to a not significant increase of particle size compared with the particle size of CPP-modified liposomes. The zeta potential of liposomes was obtained in the range of $16-22 \mathrm{mV}$, as shown in Table 1 . The TEM image of dual-modified liposomes (kFGF-Tf-lip) showed the spherical morphology of nanoparticles (Fig. 1A). Encapsulation efficiency of plasmid DNAs into liposomes was studied showing favorable plasmid encapsulation with more than $84 \%$ efficiency.

pDNA Protection Assay against Nuclease Degradation. The protective effect of liposomes on encapsulated pDNA against enzymatic degradation was evaluated incubating liposomal formulations in the presence of DNase I. Gel agarose electrophoresis (Fig. 1B) confirmed the ability of the 
liposomes to preserve the encapsulated pDNAin the presence of DNase I, whereas the naked DNA was fully digested in the same experimental conditions (Fig. 1B, lane b). There were no significant changes in the plasmid band encapsulated into liposomes (Fig. 1B, lanes e-h) compared with control (Fig. 1B, lane a), indicating that encapsulation of pDNA into liposomes protected them against enzymatic degradation.

Cytotoxicity of Liposomal Formulations. The effects of different concentrations $(100,200,400$, and $600 \mathrm{nM})$ of liposomal formulations on viability of bEnd.3 cells (Fig. 2A), primary astrocytes (Fig. 2B), and primary neurons (Fig. 2C) after 4 hours of incubation were evaluated through MTT assay. The lowest concentration $(100 \mathrm{nM})$ did not significantly affect the cell viability of the aforementioned cells, which was approximately $90 \%$. Cell viability of the cells significantly $(P<$ 0.05 ) reduced to $65 \%$ at $600 \mathrm{nM}$ phospholipid concentration of kFGF-lip, kFGF-Tf-lip, PasR8-lip, and PasR8-Tf-lip. At 600 nM phospholipid concentration, Mel-lip and Mel-Tf-lip demonstrated $57.8 \%$ of cell viabilities, which is significantly $(P<$ 0.05) less compared with kFGF-lip, kFGF-Tf-lip, PasR8-lip, and PasR8-Tf-lip at the same phospholipid concentration. Considering our findings, $100 \mathrm{nM}$ phospholipid concentration was used for further in vitro experiments because of lower cytotoxicity for all liposomal formulations.

Cellular Internalization Study. Evaluation of internalization of liposomal formulations into bEnd.3 cells, primary astrocytes, and primary neurons showed that uptake occurred in a time-dependent manner. The dual-modified liposomal formulations demonstrated $68.6 \%, 66.8 \%$, and $66.4 \%$ cellular uptake in bEnd.3 cells (Fig. 3A), primary astrocytes (Fig. 3B), and primary neurons (Fig. 3C), respectively, after 4 hours. The investigation of the mechanisms involved in liposome internalization into the cells was performed using known endocytosis inhibitors for the main endocytosis pathways. The results showed the uptake of various liposomes in bEnd.3 cells, primary astrocytes, and primary neurons after pretreatment with endocytosis inhibitors (Fig. 3, D-F). Using fluorescent-labeled liposomes, we observed differences in the mechanism of liposomes uptake dependent on cell types (Supplemental Fig. 1, A-C). Moreover, we observed that the ATP depletion induced by the energy-dependent endocytosis inhibitor sodium azide pretreatment significantly decreased the uptake of all liposomal formulations in the tested cells. Mel-lip internalization in bEnd.3 cells and primary astrocytes occurred through multiple endocytosis mechanisms, without predominance of either clathrin-mediated endocytosis, caveolae-mediated endocytosis, or macropinocytosis. Although the internalization into primary neurons preferentially occurred via caveolae-mediated endocytosis, direct translocation was also observed. Uptake of Mel-Tf-lip in bEnd.3 cells, primary astrocytes, and primary neurons was driven by multiple endocytosis pathways without significant predominance of one pathway.

Clathrin-mediated endocytosis was shown to be the main mechanism for kFGF-lip internalization into bEnd.3 cells, whereas macropinocytosis was that for primary astrocytes and primary neurons. Penetration of kFGF-Tf-lip into primary neurons occurred mainly through clathrin-mediated endocytosis, but there was no preferential route for kFGFTf-lip uptake for bEnd.3 cells and primary astrocytes. The results suggested that uptake of PasR8-lip and PasR8-Tf-lip by the aforementioned cells employs energy-dependent pathways with the involvement of clathrin-mediated endocytosis, caveolae-mediated endocytosis, and macropinocytosis.

Transfection Efficiency. To verify the potential of CPPTf-lip as an efficient gene delivery nanoplatform for treatment of neurodegenerative diseases, plasmid GFP (pGFP) and plasmid $\beta$-gal were chosen as model genes to evaluate the ability of liposomes to transfect cells. The capability of the formulations to transfect cells was compared with that of commercially available transfection agent Lipofectamine 3000. bEnd.3 cells, primary astrocytes, and primary neurons were treated with Lipofectamine 3000 or liposomal formulations containing chitosan-pGFP. After treatment, GFP expression was quantified by flow cytometer (Fig. 4, A-C) and observed using a fluorescence microscope (Fig. 4D), whereas $\beta$-galactosidase activity was measured in bEnd.3 cells, primary astrocytes, and primary neurons using a $\beta$-gal assay kit (Supplemental Fig. 2, a-c). Liposomes modified with both Tf and CPP induced higher number of cells expressing GFP compared with Lipofectamine 3000 and CPP-liposomes in all tested cells. Dual-modified liposomes demonstrated significant $(P<0.05)$ increase in the number of GFP-expressing cells (33\% in bEnd. 3 cells, $22.1 \%$ in primary astrocytes, and $18 \%$ in primary neurons) compared with Lipofectamine 3000 (10\% in bEnd.3 cells, $14 \%$ in primary astrocytes, $6.6 \%$ in primary neurons). Liposomes conjugated to $\mathrm{Tf}$ and $\mathrm{CPP}$ containing plasmid $\beta$-gal, similar to those liposomes containing pGFP, induced higher $\beta$-galactosidase activity in the tested cells compared with Lipofectamine 3000 and single-modified liposomes.

Transfection in Primary Neurons After Transport of Liposomes Across In Vitro BBB Model. The in vitro BBB model was developed by coculturing brain endothelial cells (bEnd. 3 cells) on the upper surface of culture inserts, primary astrocytes seeded on the lower surface of the culture insert, and primary neurons on the bottom of the culture wells. The integrity of this BBB model was characterized by measuring

TABLE 1

Characterization of CPP-Tf-liposomes

Data are presented as means \pm S.D. from four different preparations.

\begin{tabular}{|c|c|c|c|c|}
\hline Liposomes & $\begin{array}{c}\text { Size } \\
\mathrm{nm}\end{array}$ & PDI & $\begin{array}{l}\text { Zeta potential } \\
\mathrm{mV}\end{array}$ & $\mathrm{EE}$ \\
\hline Mel-lip & $150.9 \pm 3.39$ & $0.287 \pm 0.02$ & $20.9 \pm 2.58$ & $87.4 \% \pm 3.85 \%$ \\
\hline Mel-Tf-lip & $165.5 \pm 3.52$ & $0.175 \pm 0.05$ & $19.9 \pm 1.63$ & $84.6 \% \pm 4.89 \%$ \\
\hline kFGF-lip & $145.9 \pm 4.18$ & $0.237 \pm 0.04$ & $16.3 \pm 0.81$ & $84.5 \% \pm 1.97 \%$ \\
\hline kFGF-Tf-lip & $151.5 \pm 3.47$ & $0.192 \pm 0.03$ & $20.1 \pm 1.21$ & $90.7 \% \pm 1.96 \%$ \\
\hline PasR8-lip & $147.3 \pm 2.46$ & $0.274 \pm 0.05$ & $21.3 \pm 1.13$ & $88.2 \% \pm 7.40 \%$ \\
\hline PasR8-Tf-lip & $153.5 \pm 3.79$ & $0.181 \pm 0.01$ & $21.7 \pm 2.50$ & $90.2 \% \pm 7.30 \%$ \\
\hline
\end{tabular}

EE, pDNA entrapment efficiency; PDI, polydispersity index. 
A

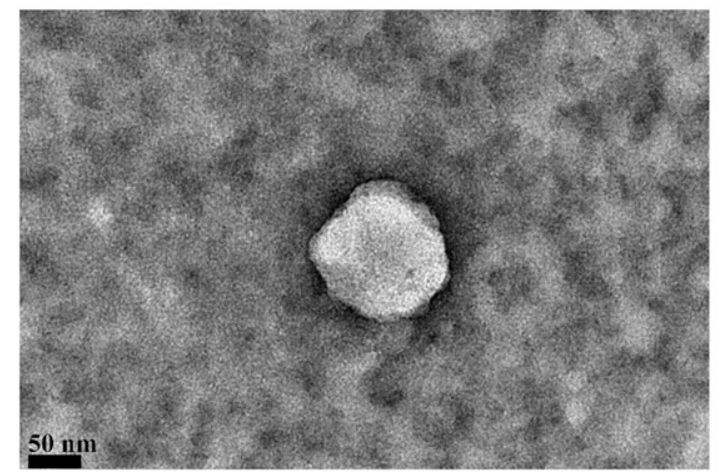

B

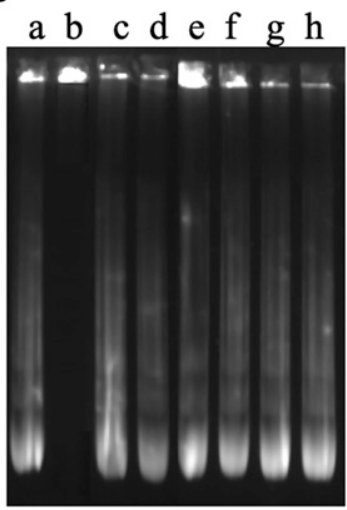

Fig. 1. (A) TEM image of kFGF-Tf-lip that were negatively stained with $0.1 \%$ phosphotungstic acid aqueous solution (scale bar, $50 \mathrm{~nm}$ ). (B) Protective effect of liposomal formulations containing chitosan-pDNA complexes against nuclease degradation. Liposomal formulation containing chitosan-pGFP (N/P 5). Lane a, naked pGFP; lane b, naked pGFP + DNase I; lanes c-h, Mel-lip, Mel-Tf-lip, kFGF-lip, kFGF-Tf-lip, PasR8-lip, and PasR8-Tf-lip containing chitosan-GFP complexes, respectively, + DNase I. the TEER and the paracellular transport of sodium fluorescein across the coculture. Using fluorescent-labeled liposomes, we showed that liposome transport across the in vitro coculture BBB model occurred over time (Fig. 5A). CPP-Tfliposomes showed superior ability to cross the in vitro $\mathrm{BBB}$ compared with CPP-liposomes. After 8 hours, approximately 10.8\% of Mel-Tf-lip, kFGF-Tf-lip, and PasR8-Tf-lip crossed the in vitro $\mathrm{BBB}$ model. The permeability coefficients of the previously mentioned formulations were 3.7-fold higher compared with that of Na-F (Fig. 5B). Despite the significantly $(P<0.05)$ higher permeability of the liposomal formulations when compared with $\mathrm{Na}-\mathrm{F}$, the values of TEER strongly indicate that liposomes did not cause membrane disruption or cellular damage after transport investigation; therefore, the integrity of the cellular barrier was maintained throughout the experiment (Fig. 5C).

Thereafter, the ability of dual-modified liposomes to transfect primary neurons after crossing the in vitro BBB model was assessed. Mel-Tf-lip, kFGF-Tf-lip, and PasR8-Tf-lip were able to induce similar expression of GFP in primary neurons (Fig. 6A), as observed in the fluorescent images of primary neurons expressing GFP after treatment with the aforementioned liposomes (Fig. 6, B-D, respectively).

Hemolytic Potential. Hemolytic potential of liposomal formulations was investigated at different phospholipid concentration (31.25-1000 $\mathrm{nM}$ ) on rat erythrocytes after 1 hour of incubation at $37^{\circ} \mathrm{C}$, as shown in Fig. 7. Similar hemolysis profile was observed for kFGF-lip, kFGF-Tf-lip, PasR8-lip, and PasR8-Tf-lip. At the lowest phospholipid concentration (31.25 nM), $1.4 \%$ of hemolysis was observed, which gradually increased to $12.2 \%$ hemolysis at $1000 \mathrm{nM}$ phospholipid concentration, whereas Mel-lip and Mel-Tf-lip at $31.25 \mu \mathrm{M}$ phospholipid concentration showed approximately $1.9 \%$ of hemolysis. These liposomal formulations significantly $(P<$ 0.05 ) increased hemolysis at $1000 \mathrm{nM}$ phospholipid concentration to $15.6 \%$. Biomaterials with hemolytic index below $2 \%$ are classified as nonhemolytic, whereas the ones within the range $2-5 \%$ are classified as slightly hemolytic and the ones above $5 \%$ are classified as hemolytic, according to the International Organization for Standardization's Standard Practice for Assessment of Hemolytic Properties of Materials (Committee F04 Medical and Surgical Materials and Devices, 2009). Therefore, liposomal formulations at low phospholipid concentration were considered nonhemolytic, which suggested them to be appropriate for intravenous administration.

In Vivo Biodistribution. Accessing the brain is one of the major hurdles faced by therapeutic agents for the treatment of central nervous system diseases. Intravenous administration of liposomal formulations intends to maximize the targeted delivery and facilitate liposomes to bypass the BBB. Then, Mel-lip, Mel-Tf-lip, kFGF-lip, kFGF-Tf-lip, PasR8-lip, and PasR8-Tf-lip were intravenously injected at a dose of 15.2 $\mu \mathrm{mol} / \mathrm{kg}$ body weight in $\mathrm{C} 57 \mathrm{BL} / 6$ mice. The evaluation of brain transport efficiency as well as the distribution in liver, kidneys, lungs, heart, spleen, and blood of fluorescentlabeled CPP-Tf-liposomes was performed after 24 hours

A

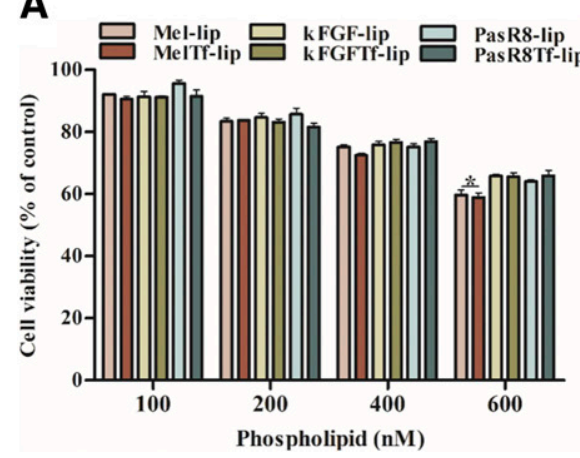

B
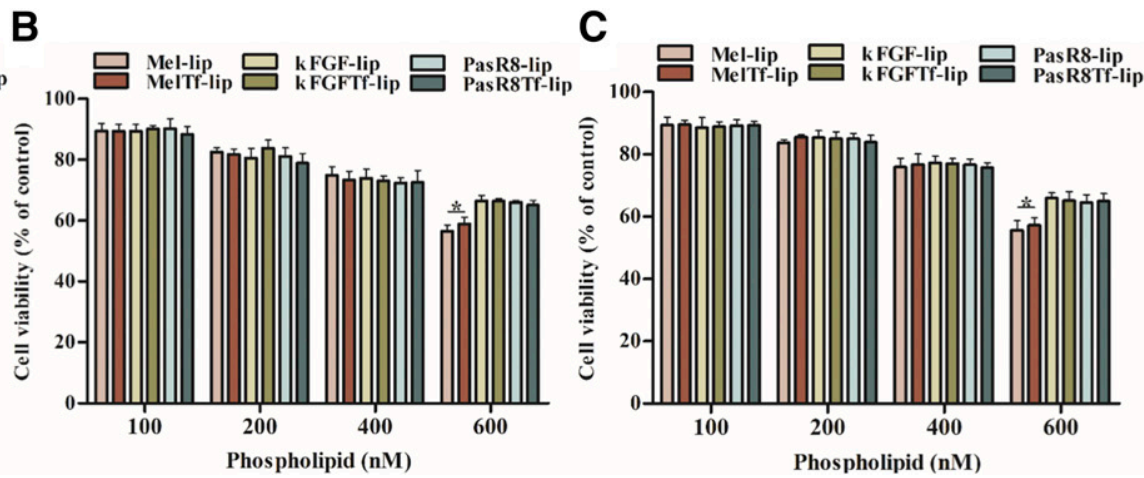

Fig. 2. In vitro viability of bEnd.3 cells (A), primary astrocytes (B), and primary neurons (C) treated with Mel-lip, Mel-Tf-lip, kFGF-lip, kFGF-Tf-lip, PasR8-lip, and PasR8-Tf-lip at different phospholipid concentrations (100, 200, 400, and $600 \mathrm{nM})$ for 4 hours. Cell viability was determined by MTT assay. Data are expressed as means \pm S.D. $(n=4) . * P<0.05$. 

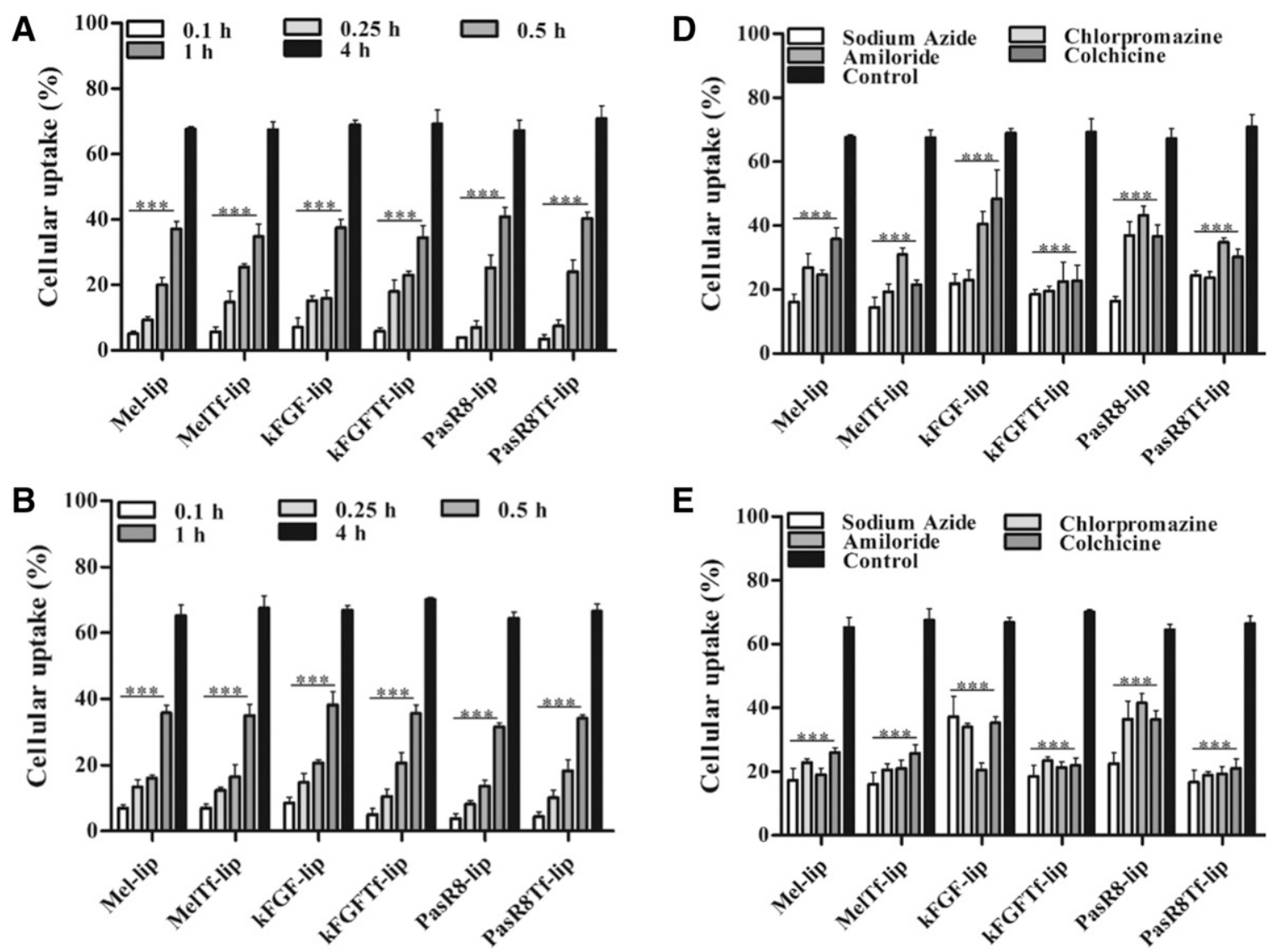

E
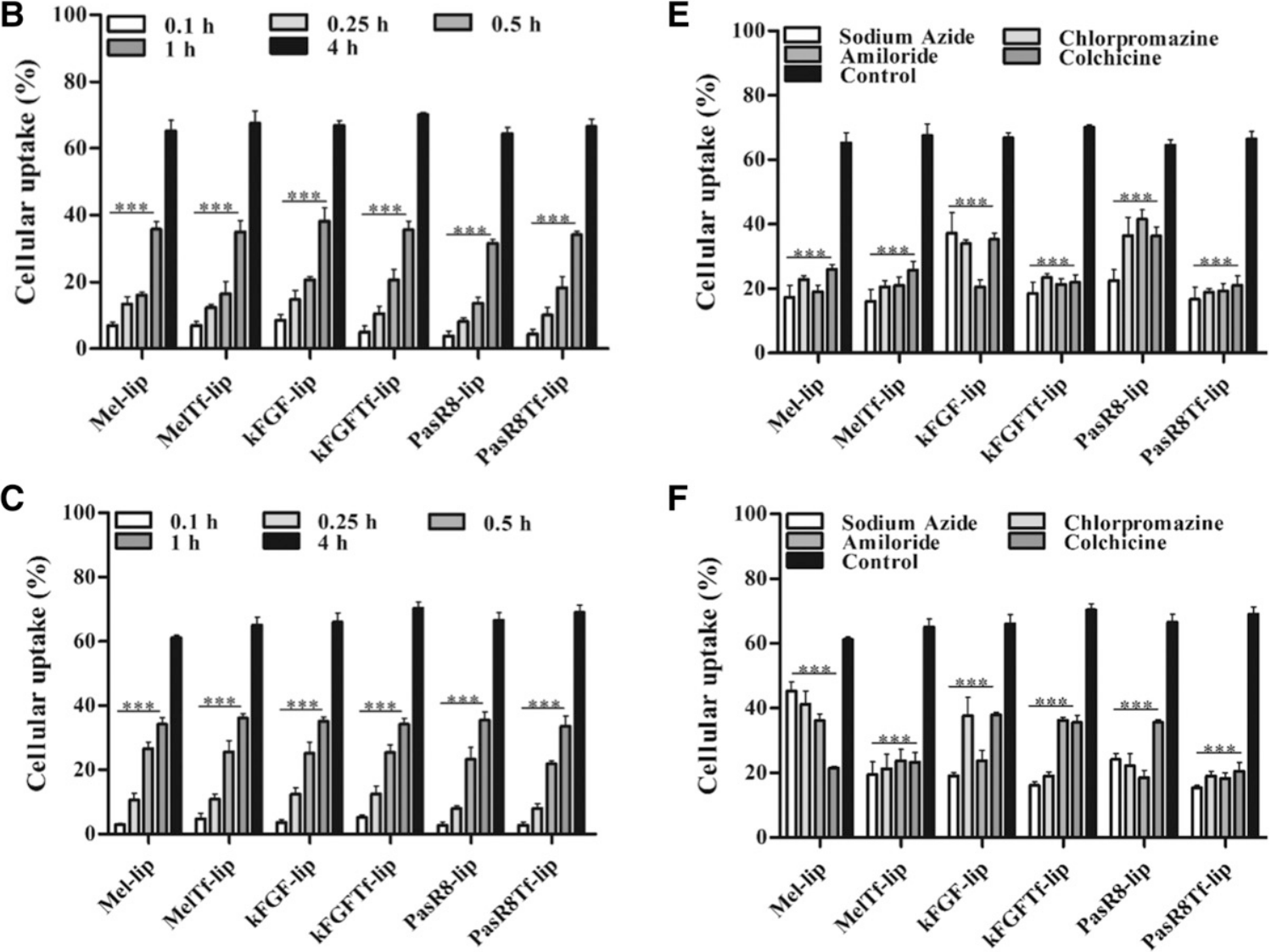

Fig. 3. Cellular uptake of Mel-lip, Mel-Tf-lip, kFGF-lip, kFGF-Tf-lip, PasR8-lip, and PasR8-Tf-lip. The amount of 1,1'-Dioctadecyl-3,3,3',3'Tetramethylindocarbocyanine Perchlorate (DiI)-labeled liposomes incorporated into bEnd.3 cells (A), primary astrocytes (B), and primary neurons (C) was measured after $0.1,0.25,0.5,1$, and 4 hours of incubation. $* * * P<0.05$ compared with liposome uptake after 4 hours of incubation. The amount of 1,1'-Dioctadecyl-3,3,3',3'-Tetramethylindocarbocyanine Perchlorate (DiI)-labeled liposomes incorporated after 4 hours of incubation into bEnd.3 cells (D), primary astrocytes (E), and primary neurons (F) pretreated with endocytosis inhibitors (sodium azide, chlorpromazine, amiloride, and colchicine). All data are expressed as means \pm S.D. $(n=4)$. $* * * P<0.05$ compared with control group $(4$ hours of incubation without endocytosis inhibitors).

following tail vein injection and expressed as percentage of the injected dose per gram of tissue (\%ID/g). The designed liposomes were able to overcome the $\mathrm{BBB}$ and permeate into the brain of mice. As shown in Fig. 8, 2.7\%, 3.2\%, 2.3\%, 5.7\%, $2.1 \%$, and $3.7 \% \mathrm{ID} / \mathrm{g}$ in the brain were quantified for Mel-lip, Mel-Tf-lip, kFGF-lip, kFGF-Tf-lip, PasR8-lip, and PasR8Tf-lip, respectively. Highlighting kFGF-Tf-lip- showed significantly $(P<0.05)$ higher ability to translocate across the $\mathrm{BBB}$ and reach the brain parenchyma compared with all liposomal formulations. Biodistribution analysis showed that liver was the major organ where liposomal formulations accumulated (average of $14.6 \% \mathrm{ID} / \mathrm{g}$ of tissue), Supplemental Fig. 3. We could observe levels of liposomal formulations between $4.8 \%$ and $10.4 \% \mathrm{ID} / \mathrm{g}$ of tissue for both kidneys and lungs, whereas $5.4 \%$ and $3.2 \% \mathrm{ID} / \mathrm{g}$ of tissue were the highest values calculate in heart and spleen, respectively, and $3.5 \% \mathrm{ID} / \mathrm{ml}$ of blood was the highest amount of liposomes quantified in the blood (Supplemental Fig. 3). Figure 8 and Supplemental Fig. 4 depict the relative fluorescent intensity of the main organs (brain, liver, kidneys, 

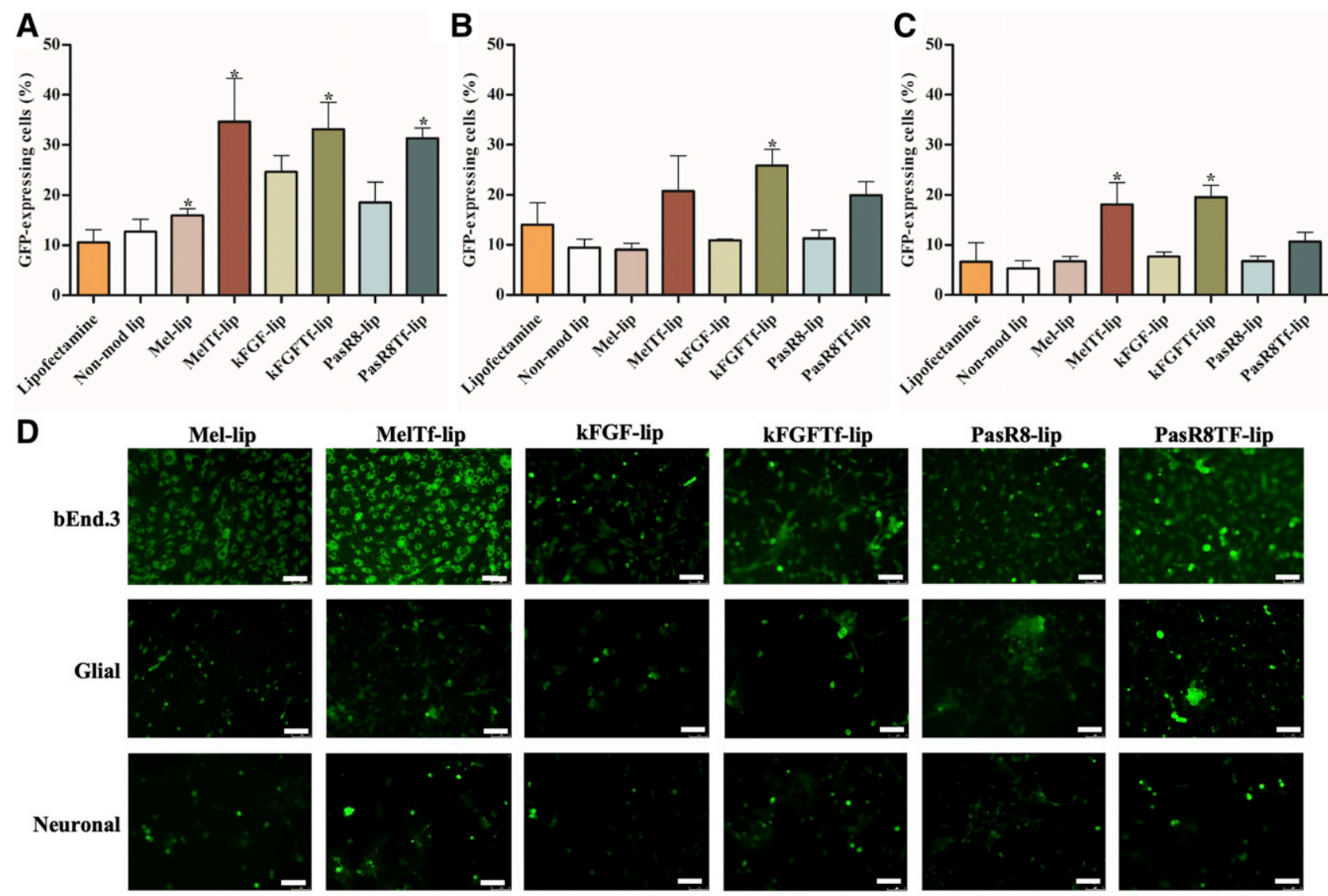

Fig. 4. GFP expression in bEnd.3 cells (A), primary astrocytes (B), and primary neurons (C) after 48 hours of treatment with Mel-lip, Mel-Tf-lip, kFGFlip, kFGF-Tf-lip, PasR8-lip, and PasR8-Tf-lip containing chitosan-pGFP complexes as determined by flow cytometry. (D) Fluorescence microscopy images of GFP expression in bEnd.3 cells, primary astrocytes, and primary neurons transfected with Mel-lip, Mel-Tf-lip, kFGF-lip, kFGF-Tf-lip, PasR8lip, and PasR8-Tf-lip containing chitosan-pGFP complexes after 48 hours (scale bar, $100 \mu \mathrm{m}$ ). Data are expressed as means \pm S.D. $(n=4)$. $* P<0.05$ compared with Lipofectamine 3000.

lungs, heart, and spleen) analyzed after 24 hours of administration with the liposomal formulations.

H\&E staining reflected the biocompatibility of liposomal formulations (Fig 9). The control group, animals administered with PBS, exhibited a healthy pattern with cellular integrity. In the treated group, animals administered with liposomal formulations, cellular damage or change in morphology was not observed. Signs of cytotoxicity including cell shrinkage, fragmentation, change in cell volume, and signs of necrosis or inflammation, were also not observed.

\section{Discussion}

Despite the advancement in therapeutic transport technology, nanoparticle formulations to treat brain diseases are still not available. Engineering liposomes based on control of
A

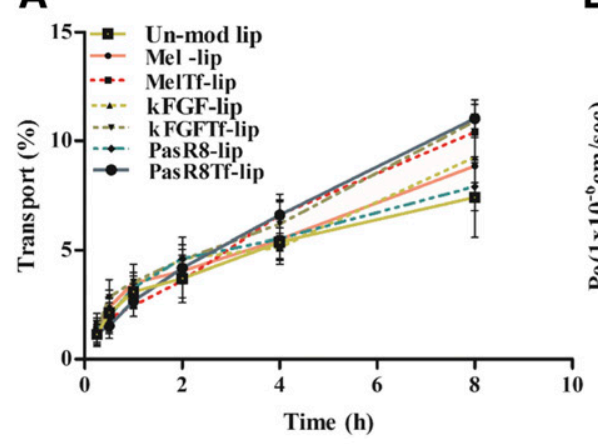

B

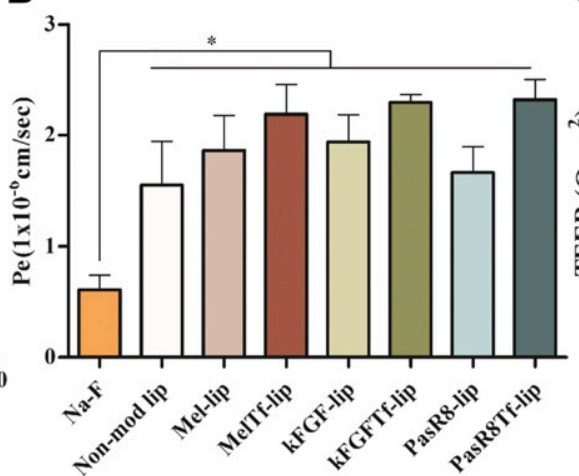

C

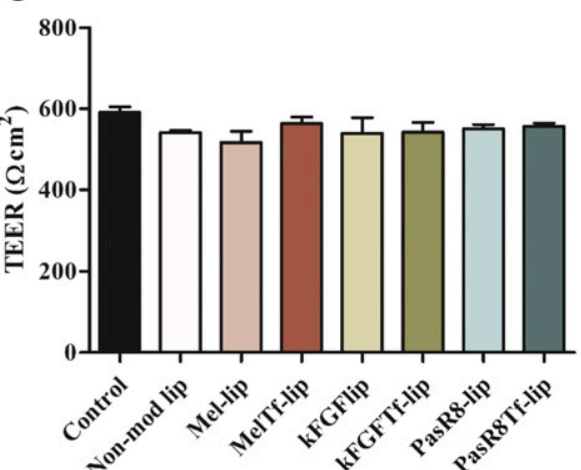

Fig. 5. (A) Percent transport of nonmodified liposome, Mel-lip, Mel-Tf-lip, kFGF-lip, kGFG-Tf-lip, PasR8-lip, and PasR8-Tf-lip across in vitro BBB model over a period of 8 hours. (B) Endothelial cell permeability (Pe, expressed as $1 \times 10^{-6} \mathrm{~cm} / \mathrm{s}$ ) coefficient for Na-F, nonmodified, Mel-lip, Mel-Tf-lip, kFGF-lip, KFGF-Tf-lip, PasR8-lip, and PasR8-Tf-lip. ${ }^{*} P<0.05$ compared with Na-F. (C) TEER (expressed as Ohm square centimeter) of in vitro BBB model before and after 8 hours of incubation with liposomal formulations. All data are expressed as means \pm S.D. $(n=4)$. 

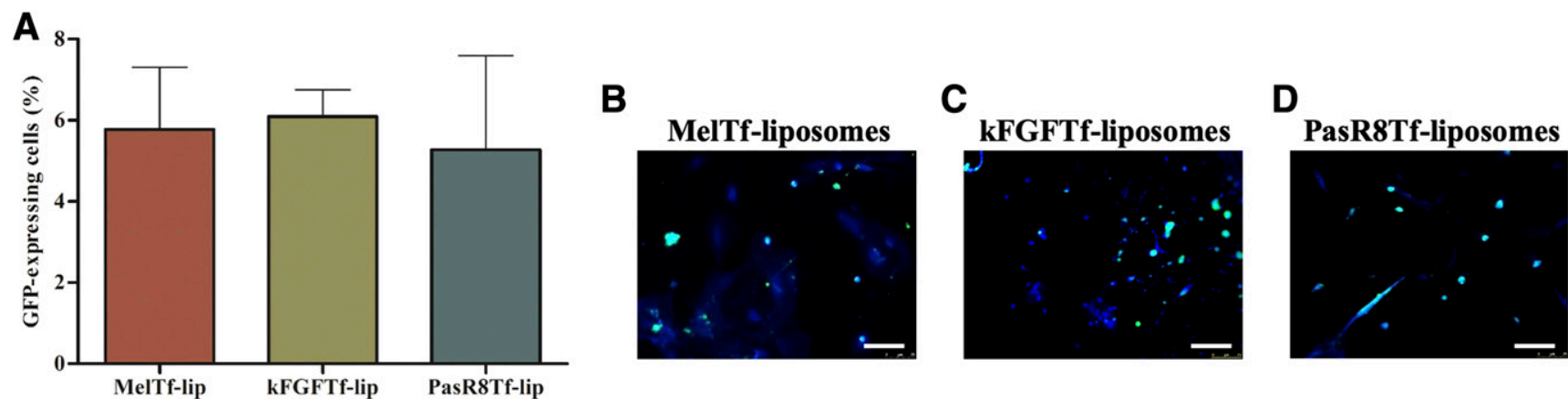

Fig. 6. (A) GFP expression in primary neurons transfected with Mel-Tf-lip, kFGF-Tf-lip, and PasR8-Tf-lip containing chitosan-GFP complexes after liposome transport through the in vitro BBB model as determined by flow cytometry. Fluorescence microscopy images of GFP expression in primary neurons transfected with Mel-Tf-lip (B), kFGF-Tf-lip (C), and PasR8-Tf-lip (D) containing chitosan-pGFP complexes after liposome transport study (scale bar, $100 \mu \mathrm{m}$ ).

composition and surface modification are being attempted to achieve targeted and delivery properties (Bunker et al., 2016; Zylberberg et al., 2017). Our group has demonstrated that the design of liposomal nanoparticles surface modified with $\mathrm{Tf}$ and cell-penetrating peptides results in efficient brain-targeted delivery systems (dos Santos Rodrigues et al., 2018, 2019b,c; Lakkadwala et al., 2019). Following the same concepts, we developed in this study liposomes modified with $\mathrm{Tf}$ and selected cell-penetrating peptides aiming to improve the delivery of plasmid DNA to brain cells after translocating the BBB. The transferrin receptors expressed on brain capillary endothelial cells help shuttle Tf-conjugated nanoparticles from blood into the brain, overcoming the impermeability of BBB. However, receptor saturation can interfere with the effects of Tf-nanoparticles and decrease their therapeutic efficacy (Johnsen and Moos, 2016; Choudhury et al., 2018). Therefore, these liposomes were surface modified with CPPs to overcome this effect.

Cell-penetrating peptides comprise a group of carriers with small peptide domains and the advantage of transporting cargo into the cells with no saturation phenomenon. In this study, we selected three CPPs (Mel, kFGF, and PasR8) based

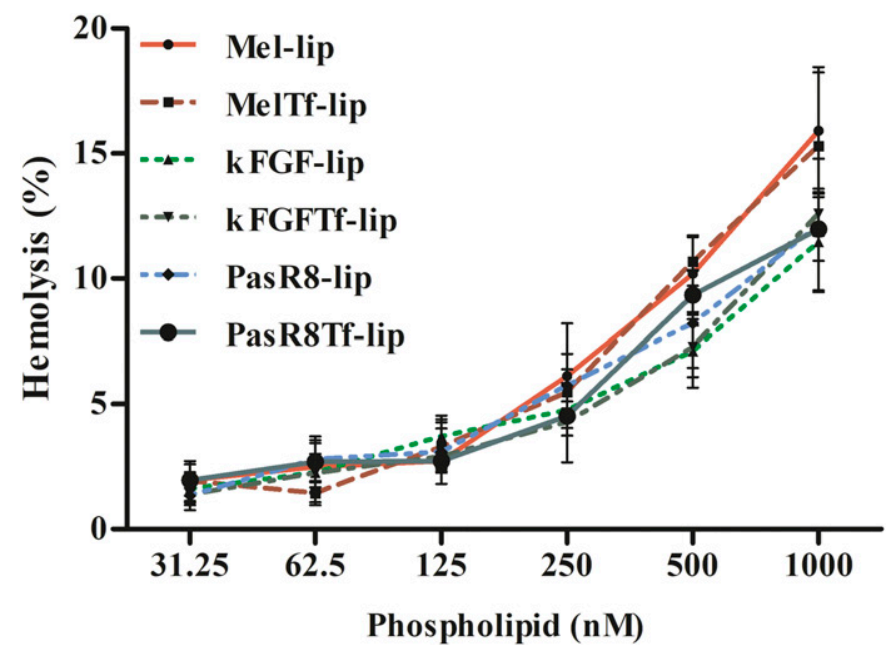

Fig. 7. Hemolytic activity of Mel-lip, Mel-Tf-lip, kFGF-lip, kFGF-Tf-lip, PasR8-lip, and PassR8-Tf-lip at different concentrations (31.25-1000 nM) in erythrocyte solution (2\%) after 1 hour of incubation at $37^{\circ} \mathrm{C}$. Hemolytic activity of $1 \% \mathrm{v} / \mathrm{v}$ Triton X-100 was considered as $100 \%$ hemolysis. Data are expressed as means \pm S.D. $(n=4)$. on their physicochemical properties and reported ability to enhance the delivery properties of conjugated drug-gene carriers (Moutal et al., 2015; Kyung et al., 2018; Zhang et al., 2018). We investigated the efficiency of liposomal
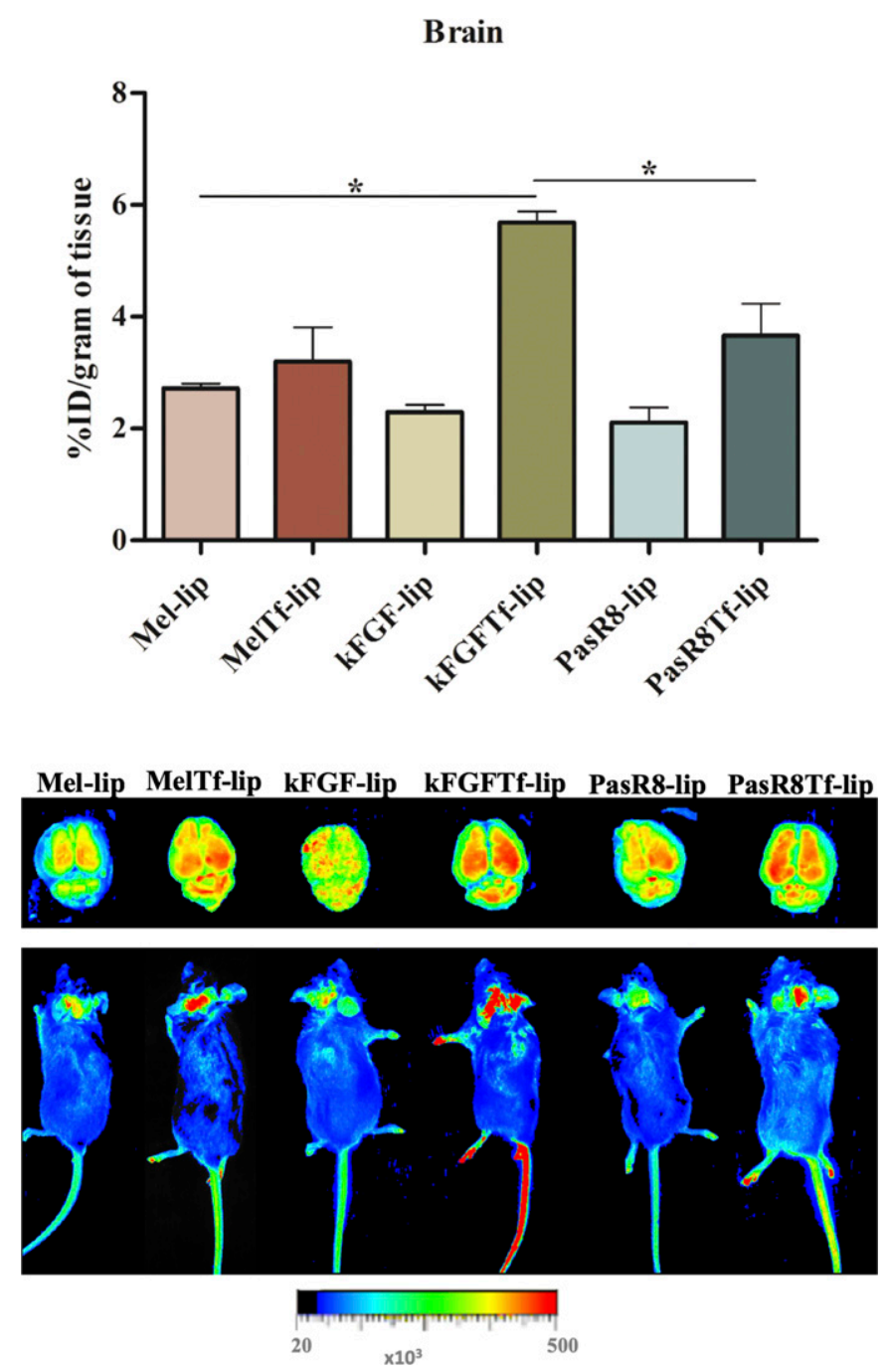

Fig. 8. In vivo biodistribution and near-infrared imaging of relative fluorescence intensity in the brain and whole mice after 24 hours of Mellip, Mel-Tf-lip, kFGF-lip, kFGF-Tf-lip, PasR8-lip, and PasR8-Tf-lip administration. Data are expressed as means \pm S.E. $(n=6)$. $* P<0.05$. 

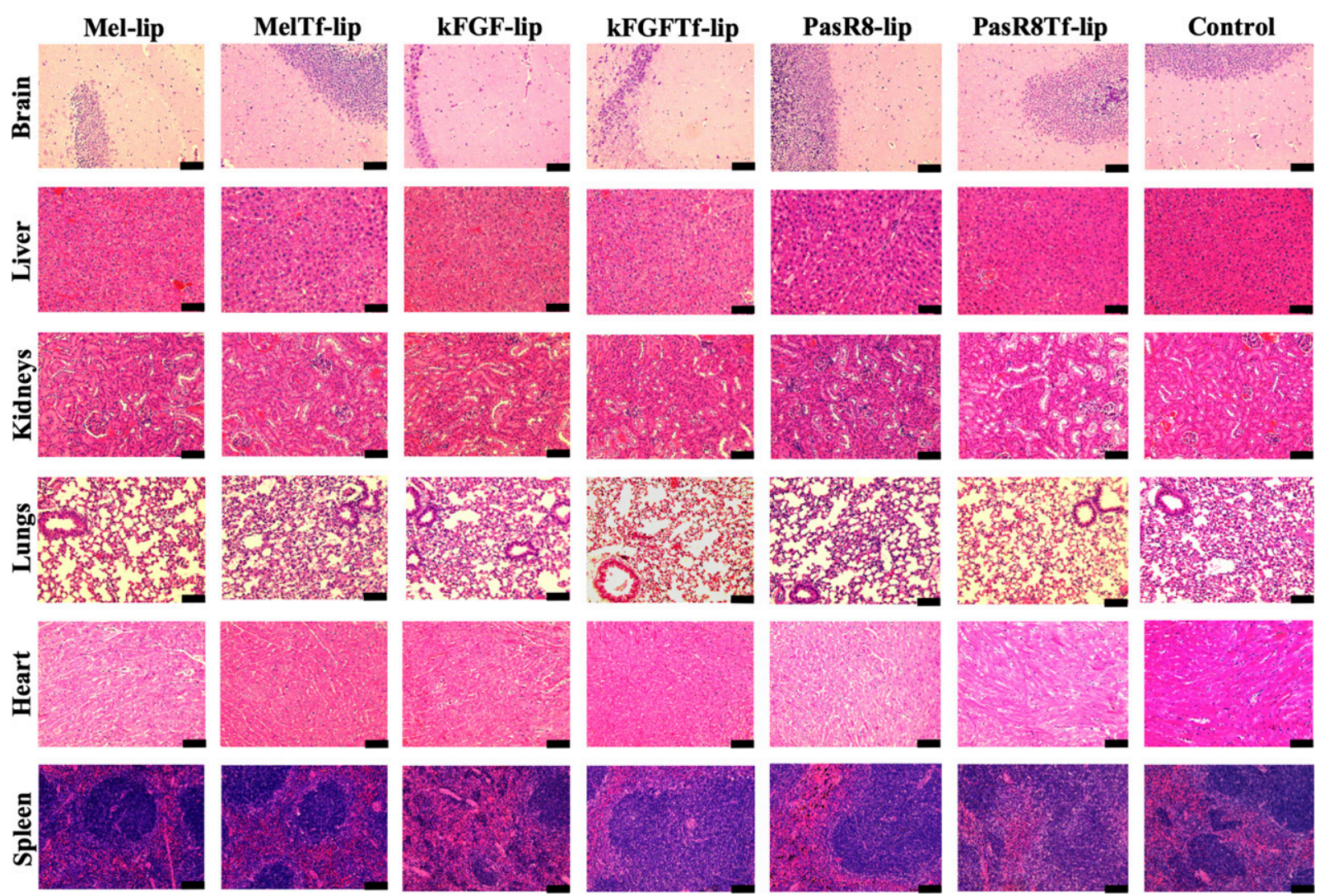

Fig. 9. Representative images of H\&E staining of brain, liver, kidneys, heart, lungs, and spleen sections of C57BL/6 mice following tail vein administration of saline (used as control), Mel-lip, Mel-Tf-lip, kFGF-lip, kFGF-Tf-lip, PasR8-lip, and PasR8-Tf-lip, $n=6$. Scale bar, $100 \mu \mathrm{m}$.

nanoparticles modified with Tf and CPP as potential braintargeted gene delivery systems.

Engineering our liposomal formulation included the lipid composition of nanoparticles, which consisted of DOTAP, DOPE, cholesterol, and DSPE-PEG. The cationic character of DOTAP likely contributed to the overall positive zeta potential of liposomal formulations. The combination of DOTAP and the helper phospholipid DOPE has been reported to cooperate with high transfection efficiency of such liposomal formulations (Hui et al., 1996; Mochizuki et al., 2013). These phospholipids would facilitate the complexation with DNA and electrostatic interaction with plasma membrane followed by release of nucleic acid for successful transfection (Ciani et al., 2004; Kim et al., 2015). DOPE can additionally contribute to endosomal escape by destabilizing endosome membrane at low pH (Balazs and Godbey, 2009). Cholesterol incorporation intended to impart nanoparticle stability and control the release of genes (Briuglia et al., 2015), and DSPEPEG would improve pharmacokinetics of liposomes after intravenous administration by minimizing protein binding and recognition of the nanoparticles by reticuloendothelial systems (Immordino et al., 2006). Complexation of plasmid DNA to chitosan was another strategy used in the design of liposomal gene delivery system with enhanced transfection efficiency. Chitosan, a cationic polymer, has extensive applications because of its versatile properties and safety. The gene delivery field has taken advantage of chitosan capacity to condense nucleic acids leading to protection against enzymatic degradation and promotion of transfection (Köping-Höggård et al., 2001).
In this study, the designed brain-targeted liposomes were prepared using thin lipid film method followed by incorporation of Tf ligand through postinsertion method. The liposomal formulations showed homogeneous particle size, which was supported by the narrow size distribution (polydispersity index $<0.3$ ). The presence of positively charged DOTAP and functional groups of Tf and CPPs may have contributed to the positive zeta potential of formulations.

Effective gene transfer is based on ensuring the delivery of the new genetic information into the target cells and the expression of the therapeutic molecule without disrupting essential regulatory functions (Ibraheem et al., 2014). However, the hydrophilic anionic nature of DNA and their susceptibility to degradation by nucleases limit their passive penetration across plasma membrane as well as their activity (Al-Dosari and Gao, 2009). Success of gene therapy depends on engineering a safe and effective carrier to ensure protection against nucleases and target delivery (Kumar et al., 2016). Our designed liposomal formulations efficiently protected the encapsulated pDNA against nuclease degradation. Such protective properties were similar to those of surface-modified liposomal formulations used in different studies (Lappalainen, 1994; Obata et al., 2009; Meissner et al., 2015).

Initially, the liposomal concentration used in our in vitro experiments was determined through evaluation of cytotoxicity in the target cells (brain endothelial cells, astrocytes, and neurons). Corroborating previous reports of our group with similar liposomal nanoparticles (dos Santos Rodrigues, 2019a,c), the formulations had negligible cytotoxicity at low phospholipid concentration. However, cell viability significantly 
reduced at high phospholipid concentration, especially for Melconjugated liposomes. This could be due to the cell lytic capabilities of Mel. Supporting our findings, Mel has shown concentration- and time-dependent cytotoxicity in studies with lymphocytes, and such properties were associated with direct membrane toxicity as well as DNA damage (Pratt et al., 2005; Lee et al., 2007). Therefore, 100 nM phospholipid concentration was chosen for the following experiments.

Better understanding of the processes that govern liposome interaction with cells, cellular internalization mechanisms, and intracellular trafficking contributes to rational design and engineering of an efficient gene delivery system (Behzadi et al., 2017). The designed liposomes entered into bEnd.3 cells, primary astrocytes, and primary neurons in a time-dependent manner. Mechanistic understanding of the biologic processes that determine the uptake of CPP-coupled liposomes as well as CPP- and Tf-coupled liposomes is important for further development of efficient gene delivery vectors with targeted properties, enhanced cellular internalization, and efficient transfection. Liposome internalization into the cells begins with interaction of the liposome moieties and plasma membrane and subsequent activation of transport pathways ((Kettler et al., 2014)Kettler et al., 2013; Behzadi et al., 2017), which may occur via either endocytosis or direct translocation through plasma membrane. We were expecting that the selected liposome moieties would trigger the activation of endocytosis through sequence-specific interaction to cellular surface. Furthermore, we were also expecting differences in liposome uptake among the tested cells because of cell-to-cell variation in plasma membrane composition including proteoglycans and receptors, which consequently may influence intracellular destination of nanoparticles (Kettler et al., 2013). Our results suggested that active endocytotic uptake mechanism was the major route for liposome internalization. The latter was in good accordance with findings on in vitro studies performed with cell-penetrating peptides (Ruzza et al., 2010; Mo et al., 2012) and surface-modified liposomes (Xu and Szoka, 1996; Miller et al., 1998; Yamano et al., 2011; Johnsen and Moos, 2016).

Mel-lip, Mel-Tf-lip, PasR8-lip, and PasR8-Tf-lip internalized into the cells through multiple endocytosis pathways, whereas kFGF-lip preferentially internalized into bEnd.3 cells via clathrin-mediated endocytosis, and astrocytes and neurons via macropinocytosis. Clathrin-mediated endocytosis was the main mechanism for kFGF-Tf-lip to internalize into primary neurons. The translocation potential of $\mathrm{kFGF}$ conjugated cargos has been suggested to be related to the overall hydrophobic composition of the peptide (Moutal et al., 2015). Our findings are in accordance with earlier reports showing that internalization of hydrophobic CPPs into cells happened mainly via endocytotic pathway (Madani et al., 2011). Similarly, the promotion of PasR8 or peptide-modified nanoparticles translocation into cytoplasm was observed to be predominantly an energy-dependent mechanism in different studies (Takayama et al., 2009, 2012; Liu et al., 2013). It suggested that mechanism was based on destabilization of plasma membrane during early stages of endocytosis. The hydrophobic segment Pas attached to R8 facilitated the peptide-proteoglycan interactions, thereby enhancing their internalization (Takayama et al., 2009, 2012).

In vitro transfection studies showed that liposomal formulations dual-modified with Tf and CPP had superior ability to deliver pDNA into cells and induce higher protein expression when compared with CPP-liposomes and Lipofectamine. Our findings are in accordance with studies that demonstrated the enhancement in transfection provided by liposome modified with specific ligands (Zou et al., 1999; Meissner et al., 2015; Zylberberg et al., 2017). Additionally, combination with transferrin receptor targeting has become a consistent strategy for both targeting properties and enhanced transfection (Cheng, 1996; da Cruz et al., 2004, 2005).

A significant number of potential formulations for the treatment of central nervous system diseases fail to bypass the BBB. Therefore, it is relevant to characterize BBB permeability of these candidates in a reliable in vitro $\mathrm{BBB}$ model. This system can provide a better understanding of mechanisms involved in crossing such a tight barrier and can allow screening as well as optimization of candidate formulations (Hatherell et al., 2011; Helms et al., 2016). The designed coculture BBB model provides close resemblance to the cell arrangement at the neurovasculature unit and therefore it has become a more widely accepted model (Wilhelm and Krizbai, 2014). The transport of liposomal formulations across the in vitro $\mathrm{BBB}$ occurred over time without disrupting the barrier layer, which correlates to the observed low cytotoxicity of liposomal formulations. Furthermore, the results suggest that transferrin receptor targeting facilitated in interaction of CPP-Tf-liposomes to Tf receptor on cell surface enhancing the transport across the in vitro barrier layer. The translocation of Tf-coupled liposomes suggested the involvement of clathrinmediated transcytosis in this process.

Concerns about the biologic safety of cationic nanoparticles administered intravenously need to be addressed prior in vivo application. The interaction between biomaterial and erythrocytes was evaluated using hemolysis assay. The hemolytic profile of liposomal formulations showed that hemolysis increased as phospholipid concentration increased. At low phospholipid concentration, hemolysis index was in the range classified as nonhemolytic (Committee F04 Medical and Surgical Materials and Devices, 2009). At higher phospholipid concentration, the superior levels of hemolysis induced by Mel-lip and Mel-Tf-lip could be attributed to the strong interaction of Mel with cell membranes, which can cause disruption of lipid structure accounting for erythrocyte lysis. Membrane binding and lysis properties of Mel depend not only on the plasma membrane composition but also on peptide concentration (Lundquist et al., 2008; Qian and Heller, 2015). Furthermore, conjugation to DSPE-PEG has been demonstrated to reduce the lytic activity of Mel (Popplewell et al., 2007). Taken together, these could be an explanation for the lower levels of hemolysis induced by Mel-lip and Mel-Tf-lip at low phospholipid concentration. The study of direct effects of Mel on erythrocytes exposed to Mel concentrations of $0.5,0.75$, and $1 \mu \mathrm{M}$ demonstrated the lytic properties of this peptide, which produced variation on median cell volume followed by cell lysis (Pratt et al., 2005). The low hemolytic index at low phospholipid concentration indicated that our designed liposomes are appropriate for systemic administration.

The qualitative and quantitative evaluation of biodistribution of our brain-targeted liposomes showed the ability of the nanoparticles to overcome the BBB and dual modification with both ligands enhancing the brain-targeting ability. Liposomes modified with kFGF and Tf showed significantly $(P<0.05)$ higher accumulation in the brain compared with Mel-Tf-lip and 
PasR8-Tf-lip. Physicochemical properties of kFGF might have contributed to enhancement of transport of modified liposomes across BBB. The latter may be attributed to the reported high translocation across BBB with reduced efflux from the brain and longer half-life ( 48 hours) of kFGF compared with Mel and arginine-rich peptides (4 minutes and 2 hours, respectively) (Sarko et al., 2010; Moutal et al., 2015). Additional studies are needed to investigate the mechanisms involved in nanoparticle transcytosis across BBB, delivery of pDNA, and induction of protein expression in brain cells. Liposomal formulations were also detected in other organs and accumulated more in liver. Many research groups have developed advanced brain delivery carriers based on liposomes coated with specific ligands that ensure brain targeting and prolonged systemic circulation properties (Qin et al., 2011; Zheng et al., 2015; Gregori et al., 2016; Ordóñez-Gutiérrez et al., 2017; Choudhury et al., 2018). The brain-targeted properties and accumulation of the designed liposomes combining transferrin receptor targeting and enhanced cell penetration in our study showed advantageous application as brain gene carrier. Furthermore, the overexpression of transferrin receptor on brain capillary endothelium could strength the targeting ability of dualfunctionalized liposomes especially of kFGF-Tf-lip. This finding followed the similar efficiency of liposomes to accumulate in the brain described in earlier reports of our group, suggesting the importance of specific ligands enhancing the transport of multifunctionalized liposomes across BBB (dos Santos Rodrigues et al., 2018, 2019c). Additionally, these studies also suggested that physicochemical properties of coupled CPPs determined the differences in the ability of liposomes to reach the brain. Studies characterizing the role of CPPs on in vivo transport of designed liposomes across BBB may explain the differences in transport efficiency of formulations across the BBB and, consequently, in transfection efficiency.

The biologically inspired lipid-based nanoparticles, conjugated to Tf ligand and CPP, were here designed as a novel nanomedicine for efficient delivery of genes into the brain. Both in vitro and in vivo experiments evaluated the ability of these liposomes to translocate across cell membrane and transfect cells. We could note that the physicochemical properties of the selected CPPs (Mel, kFGF, and PasR8) influenced the ability of the designed liposomes to internalize and transfect cells as well as to cross in vitro and in vivo $\mathrm{BBB}$ and penetrate into the brain. The established in vitro coculture BBB model was shown to be a reliable model to estimate the ability of the delivery systems to penetrate into the central nervous system. Liposomes conjugated to $\mathrm{Tf}$ and kFGF showed significantly higher ability to overcome BBB and reach the brain of mice compared with the other dualfunctionalized liposomes. The findings here provided evidence that strategic design of liposomal formulations might serve as efficient approach to obtain delivery system with desired properties. Importantly, the data also highlight the importance for strategic design of liposomal formulations involving the use of transferrin receptor targeting and CPPs to improve carrier gene delivery properties prior to their translation in vivo.

\section{Authorship Contributions}

Participated in research design: dos Santos Rodrigues, Lakkadwala, Kanekiyo, Singh.

Conducted experiments: dos Santos Rodrigues.
Performed data analysis: dos Santos Rodrigues.

Wrote and contributed to the writing of the manuscript: dos Santos Rodrigues, Lakkadwala, Singh.

\section{References}

Al-Dosari MS and Gao X (2009) Nonviral gene delivery: principle, limitations, and recent progress. AAPS J 11:671-681.

Balazs DA and Godbey WT (2009) Liposomes for use in gene delivery. Biomacromoles 10:2379-2400.

Behzadi S, Serpooshan V, Tao W, Hamaly MA, Alkawareek MY, Dreaden EC, Brown D, Alkilany AM, Farokhzad OC, and Mahmoudi M (2017) Cellular uptake of nanoparticles: journey inside the cell. Chem Soc Rev 46:4218-4244.

Bolhassani A, Jafarzade BS, and Mardani G (2017) In vitro and in vivo delivery of therapeutic proteins using cell penetrating peptides. Peptides 87:50-63.

Briuglia M-L, Rotella C, McFarlane A, and Lamprou DA (2015) Influence of cholesterol on liposome stability and on in vitro drug release. Drug Deliv Transl Res 5: 231-242.

Bunker A, Magarkar A, and Viitala T (2016) Rational design of liposomal drug delivery systems, a review: combined experimental and computational studies of lipid membranes, liposomes and their PEGylation. Biochim Biophys Acta 1858: $2334-2352$

Cai D, Gao W, He B, Dai W, Zhang H, Wang X, Wang J, Zhang X, and Zhang Q (2014) Hydrophobic penetrating peptide PFVYLI-modified stealth liposomes for doxorubicin delivery in breast cancer therapy. Biomaterials 35:2283-2294.

Cheng P-W (1996) Receptor ligand-facilitated gene transfer: enhancement of liposome-mediated gene transfer and expression by transferrin. Hum Gene Ther $\mathbf{7}$ $275-282$

Choudhury H, Pandey M, Chin PX, Phang YL, Cheah JY, Ooi SC, Mak K-K, Pichika MR, Kesharwani P, Hussain Z, et al. (2018) Transferrin receptors-targeting nanocarriers for efficient targeted delivery and transcytosis of drugs into the brain tumors: a review of recent advancements and emerging trends. Drug Deliv Transt Res 8:1545-1563.

Ciani L, Ristori S, Salvati A, Calamai L, and Martini G (2004) DOTAP/DOPE and DC-Chol/DOPE lipoplexes for gene delivery: zeta potential measurements and electron spin resonance spectra. Biochim Biophys Acta 1664:70-79.

Committee F04 Medical and Surgical Materials and Devices (2009) Standard practice for assessment of hemolytic properties of materials. ASTM International, West Conshohocken, PA.

Cook RL, Householder KT, Chung EP, Prakapenka AV, DiPerna DM, and Sirianni RW (2015) A critical evaluation of drug delivery from ligand modified nanoparticles: confounding small molecule distribution and efficacy in the central nervous system. J Control Release 220 (Pt A):89-97.

Costantini LC, Bakowska JC, Breakefield XO, and Isacson O (2000) Gene therapy in the CNS. Gene Ther 7:93-109.

da Cruz MT, Cardoso ALC, de Almeida LP, Simões S, and de Lima MC (2005) Tflipoplex-mediated NGF gene transfer to the CNS: neuronal protection and recovery in an excitotoxic model of brain injury. Gene Ther 12:1242-1252.

da Cruz MT, Simões S, and de Lima MC (2004) Improving lipoplex-mediated gene transfer into $\mathrm{C} 6$ glioma cells and primary neurons. Exp Neurol 187: 65-75.

de Figueiredo IR, Freire JM, Flores L, Veiga AS, and Castanho MARB (2014) Cellpenetrating peptides: a tool for effective delivery in gene-targeted therapies. IUBMB Life 66:182-194.

Dos Santos Rodrigues B, Banerjee A, Kanekiyo T, and Singh J (2019a) Functionalized liposomal nanoparticles for efficient gene delivery system to neuronal cell transfection. Int J Pharm 566:717-730.

Dos Santos Rodrigues B, Kanekiyo T, and Singh J (2019b) ApoE-2 brain-targeted gene therapy through transferrin and penetratin tagged liposomal nanoparticles. Pharm Res 36:161.

Dos Santos Rodrigues B, Lakkadwala S, Kanekiyo T, and Singh J (2019c) Development and screening of brain-targeted lipid-based nanoparticles with enhanced cell penetration and gene delivery properties. Int $J$ Nanomedicine 14: $6497-6517$

Dos Santos Rodrigues B, Oue H, Banerjee A, Kanekiyo T, and Singh J (2018) Dual functionalized liposome-mediated gene delivery across triple co-culture blood brain barrier model and specific in vivo neuronal transfection. $J$ Control Release 286: 264-278.

Gregori M, Taylor M, Salvati E, Re F, Mancini S, Balducci C, Forloni G, Zambelli V, Sesana S, Michael M, et al. (2016) Retro-inverso peptide inhibitor nanoparticles as potent inhibitors of aggregation of the Alzheimer's A $\beta$ peptide. Nanomedicine 13: $723-732$.

Hatherell K, Couraud PO, Romero IA, Weksler B, and Pilkington GJ (2011) Development of a three-dimensional, all-human in vitro model of the blood-brain barrier using mono-, co-, and tri-cultivation Transwell models. J Neurosci Methods 199:223-229.

Helms HC, Abbott NJ, Burek M, Cecchelli R, Couraud P-O, Deli MA, Förster C, Galla HJ, Romero IA, Shusta EV, et al. (2016) In vitro models of the blood-brain barrier: an overview of commonly used brain endothelial cell culture models and guidelines for their use. J Cereb Blood Flow Metab 36:862-890.

Hui SW, Langner M, Zhao YL, Ross P, Hurley E, and Chan K (1996) The role of helper lipids in cationic liposome-mediated gene transfer. Biophys $J \mathbf{7 1}$ $590-599$.

Ibraheem D, Elaissari A, and Fessi H (2014) Gene therapy and DNA delivery systems. Int $J$ Pharm 459:70-83.

Johnsen KB and Moos T (2016) Revisiting nanoparticle technology for blood-brain barrier transport: unfolding at the endothelial gate improves the fate of transferrin receptor-targeted liposomes. J Control Release 222:32-46.

Kang YJ, Cutler EG, and Cho H (2018) Therapeutic nanoplatforms and delivery strategies for neurological disorders. Nano Converg 5:35. 
Kettler K, Veltman K, van de Meent D, van Wezel A, and Hendriks AJ (2014) Cellular uptake of nanoparticles as determined by particle properties, experimental conditions, and cell type. Environ Toxicol Chem 33:481-492.

Khalil IA, Kogure K, Futaki S, Hama S, Akita H, Ueno M, Kishida H, Kudoh M, Mishina Y, Kataoka K, et al. (2007) Octaarginine-modified multifunctional envelope-type nanoparticles for gene delivery. Gene Ther 14:682-689.

Khalil IA, Kogure K, Futaki S, and Harashima H (2006) High density of octaarginine stimulates macropinocytosis leading to efficient intracellular trafficking for gene expression. J Biol Chem 281:3544-3551.

Kim BK, Hwang GB, Seu YB, Choi JS, Jin KS, and Doh KO (2015) DOTAP/DOPE ratio and cell type determine transfection efficiency with DOTAP-liposomes. Biochim Biophys Acta 1848 (10 Pt A):1996-2001.

Kim HK, Davaa E, Myung CS, and Park JS (2010) Enhanced siRNA delivery using cationic liposomes with new polyarginine-conjugated PEG-lipid. Int J Pharm 392 141-147.

Köping-Höggård M, Tubulekas I, Guan H, Edwards K, Nilsson M, Vårum KM, and Artursson P (2001) Chitosan as a nonviral gene delivery system. Structureproperty relationships and characteristics compared with polyethylenimine in vitro and after lung administration in vivo. Gene Ther 8:1108-1121.

Kristensen M, Birch D, and Mørck Nielsen H (2016) Applications and challenges for use of cell-penetrating peptides as delivery vectors for peptide and protein cargos. Int J Mol Sci 17:185.

Krukemeyer MG, Krenn V, and Huebner F (2015) History and possible uses of nanomedicine based on nanoparticles and nanotechnological progress. J Nanomed Nanotechnol 6:6.

Kumar SR, Markusic DM, Biswas M, High KA, and Herzog RW (2016) Clinical development of gene therapy: results and lessons from recent successes. Mol Ther Methods Clin Dev 3:16034.

Kyung H, Kim H, Lee H, and Lee SJ (2018) Enhanced intracellular delivery of macromolecules by melittin derivatives mediated cellular uptake. J Ind Eng Chem 58:290-295.

Lakkadwala S, Dos Santos Rodrigues B, Sun C, and Singh J (2019) Dual functionalized liposomes for efficient co-delivery of anti-cancer chemotherapeutics for the treatment of glioblastoma. $J$ Control Release 307:247-260.

Lappalainen K (1994) Cationic liposomes improve stability and intracellular delivery of antisense oligonucleotides into CaSki cells. Biophysica 1196:201-208.

Layek B, Haldar MK, Sharma G, Lipp L, Mallik S, and Singh J (2014) Hexanoic acid and polyethylene glycol double grafted amphiphilic chitosan for enhanced gene delivery: influence of hydrophobic and hydrophilic substitution degree. Mol Pharm 11:982-994.

Lee YJ, Kang SJ, Kim BM, Kim YJ, Woo HD, and Chung HW (2007) Cytotoxicity of honeybee (Apis mellifera) venom in normal human lymphocytes and HL-60 cells Chem Biol Interact 169:189-197.

Li SD and Huang L (2010) Stealth nanoparticles: high density but sheddable PEG is a key for tumor targeting. J Control Release 145:178-181.

Li X, Ding L, Xu Y, Wang Y, and Ping Q (2009) Targeted delivery of doxorubicin using stealth liposomes modified with transferrin. Int J Pharm 373:116-123.

Lin YZ, Yao S, Veach RA, Torgerson TR, and Hawiger J (1995) Inhibition of nuclear translocation of transcription factor NF-kappa B by a synthetic peptide containing a cell membrane-permeable motif and nuclear localization sequence. J Biol Chem 270:14255-14258.

Liu BR, Lo SY, Liu CC, Chyan CL, Huang YW, Aronstam RS, and Lee HJ (2013) Endocytic trafficking of nanoparticles delivered by cell-penetrating peptides comprised of nona-arginine and a penetration accelerating sequence. PLoS One 8:e67100.

Liu C, Liu XN, Wang GL, Hei Y, Meng S, Yang LF, Yuan L, and Xie Y (2017) A dualmediated liposomal drug delivery system targeting the brain: rational construction, integrity evaluation across the blood-brain barrier, and the transporting mechanism to glioma cells. Int $J$ Nanomedicine 12:2407-2425.

Lundquist A, Wessman P, Rennie AR, and Edwards K (2008) Melittin-lipid interaction: a comparative study using liposomes, micelles and bilayer disks. Biochim Biophys Acta 1778:2210-2216.

Immordino ML, Dosio F, and Cattel L (2006) Stealth liposomes: review of the basic science, rationale, and clinical applications, existing and potential. Int $J$ Nanomedicine 1:297-315.

Madani F, Lindberg S, Langel U, Futaki S, and Gräslund A (2011) Mechanisms of cellular uptake of cell-penetrating peptides. J Biophys 2011:414729.

Meissner JM, Toporkiewicz M, Czogalla A, Matusewicz L, Kuliczkowski K and Sikorski AF (2015) Novel antisense therapeutics delivery systems: in vitro and in vivo studies of liposomes targeted with anti-CD20 antibody. $J$ Control Release 220 (Pt A):515-528.

Miller CR, Bondurant B, McLean SD, McGovern KA and O'Brien DF (1998) Liposome-cell interactions in vitro: effect of liposome surface charge on the binding and endocytosis of conventional and sterically stabilized liposomes. Biochemistry 37:12875-12883.

Mo RH, Zaro JL, and Shen WC (2012) Comparison of cationic and amphipathic cell penetrating peptides for siRNA delivery and efficacy. Mol Pharm 9:299-309.

Mochizuki S, Kanegae N, Nishina K, Kamikawa Y, Koiwai K, Masunaga H, and Sakurai K (2013) The role of the helper lipid dioleoylphosphatidylethanolamine (DOPE) for DNA transfection cooperating with a cationic lipid bearing ethylenediamine. Biochim Biophys Acta 1828:412-418.

Moutal A, François-Moutal L, Brittain JM, Khanna M, and Khanna R (2015) Differential neuroprotective potential of CRMP2 peptide aptamers conjugated to cationic, hydrophobic, and amphipathic cell penetrating peptides. Front Cell Neurosci 8:471.

Nag OK and Awasthi V (2013) Surface engineering of liposomes for stealth behavior. Pharmaceutics 5:542-569.

Nakagawa S, Deli MA, Kawaguchi H, Shimizudani T, Shimono T, Kittel A, Tanaka $\mathrm{K}$, and Niwa M (2009) A new blood-brain barrier model using primary rat brain endothelial cells, pericytes and astrocytes. Neurochem Int 54:253-263.

Niu X, Chen J, and Gao J (2019) Nanocarriers as a powerful vehicle to overcome blood-brain barrier in treating neurodegenerative diseases: focus on recent advances. Asian J Pharm Sci 14:480-496.
Obata Y, Saito S, Takeda N, and Takeoka S (2009) Plasmid DNA-encapsulating liposomes: effect of a spacer between the cationic head group and hydrophobic moieties of the lipids on gene expression efficiency. Biochim Biophys Acta 1788:1148-1158.

Ordóñez-Gutiérrez L, Posado-Fernández A, Ahmadvand D, Lettiero B, Wu L, Antón M, Flores O, Moghimi SM, and Wandosell F (2017) ImmunoPEGliposomemediated reduction of blood and brain amyloid levels in a mouse model of Alzheimer's disease is restricted to aged animals. Biomaterials 112:141-152.

Pino-Angeles A and Lazaridis T (2018) Effects of peptide charge, orientation, and concentration on melittin transmembrane pores. Biophys $J$ 114:2865-2874.

Popplewell JF, Swann MJ, Freeman NJ, McDonnell C, and Ford RC (2007) Quantifying the effects of melittin on liposomes. Biochim Biophys Acta 1768:13-20.

Pratt JP, Ravnic DJ, Huss HT, Jiang X, Orozc BS, and Mentzer SJ (2005) Melittininduced membrane permeability: a nonosmotic mechanism of cell death. In Vitro Cell Dev Biol Anim 41:349-355.

Qian S and Heller WT (2015) Melittin-induced cholesterol reorganization in lipid bilayer membranes. Biochim Biophys Acta 1848 (10 Pt A):2253-2260.

Qin Y, Chen H, Zhang Q, Wang X, Yuan W, Kuai R, Tang J, Zhang L, Zhang Z, Zhang $\mathrm{Q}$, et al. (2011) Liposome formulated with TAT-modified cholesterol for improving brain delivery and therapeutic efficacy on brain glioma in animals. Int J Pharm 420:304-312.

Ramsey JD and Flynn NH (2015) Cell-penetrating peptides transport therapeutics into cells. Pharmacol Ther 154:78-86.

Ruzza P, Biondi B, Marchiani A, Antolini N, and Calderan A (2010) Cell-penetrating peptides: a comparative study on lipid affinity and cargo delivery properties. Pharmaceuticals (Basel) 3:1045-1062.

Saeedi M, Eslamifar M, Khezri K, and Dizaj SM (2019) Applications of nanotechnology in drug delivery to the central nervous system. Biomed Pharmacother 111:666-675. Saffari M, Moghimi HR, and Dass CR (2016) Barriers to liposomal gene delivery: from application site to the target. Iran J Pharm Res 15 (Suppl):3-17.

Sarko D, Beijer B, Garcia Boy R, Nothelfer EM, Leotta K, Eisenhut M, Altmann A, Haberkorn U, and Mier W (2010) The pharmacokinetics of cell-penetrating peptides. Mol Pharm 7:2224-2231.

Sharma G, Modgil A, Layek B, Arora K, Sun C, Law B, and Singh J (2013) Cell penetrating peptide tethered bi-ligand liposomes for delivery to brain in vivo: biodistribution and transfection. J Control Release 167:1-10.

Sharma G, Modgil A, Sun C, and Singh J (2012) Grafting of cell-penetrating peptide to receptor-targeted liposomes improves their transfection efficiency and transport across blood-brain barrier model. J Pharm Sci 101:2468-2478.

Sharma G, Modgil A, Zhong T, Sun C, and Singh J (2014) Influence of short-chain cell-penetrating peptides on transport of doxorubicin encapsulating receptortargeted liposomes across brain endothelial barrier. Pharm Res 31:1194-1209.

Sumners C and Fregly MJ (1989) Modulation of angiotensin II binding sites in neuronal cultures by mineralocorticoids. Am J Physiol 256:C121-C129.

Takayama K, Hirose H, Tanaka G, Pujals S, Katayama S, Nakase I, and Futaki S (2012) Effect of the attachment of a penetration accelerating sequence and the influence of hydrophobicity on octaarginine-mediated intracellular delivery. $\mathrm{Mol}$ Pharm 9:1222-1230.

Takayama K, Nakase I, Michiue H, Takeuchi T, Tomizawa K, Matsui H, and Futaki S (2009) Enhanced intracellular delivery using arginine-rich peptides by the addition of penetration accelerating sequences (Pas). J Control Release 138:128-133.

Upadhya A and Sangave PC (2016) Hydrophobic and electrostatic interactions between cell penetrating peptides and plasmid DNA are important for stable noncovalent complexation and intracellular delivery. J Pept Sci 22:647-659.

Wang B, He C, Tang C, and Yin C (2011) Effects of hydrophobic and hydrophilic modifications on gene delivery of amphiphilic chitosan based nanocarriers. Biomaterials 32:4630-4638.

Wilhelm I and Krizbai IA (2014) In vitro models of the blood-brain barrier for the study of drug delivery to the brain. Mol Pharm 11:1949-1963.

Xu Y and Szoka FC Jr. (1996) Mechanism of DNA release from cationic liposome/ DNA complexes used in cell transfection. Biochemistry 35:5616-5623.

Yamano S, Dai J, Yuvienco C, Khapli S, Moursi AM, and Montclare JK (2011) Modified Tat peptide with cationic lipids enhances gene transfection efficiency via temperaturedependent and caveolae-mediated endocytosis. J Control Release 152:278-285.

Yameen B, Choi WI, Vilos C, Swami A, Shi J, and Farokhzad OC (2014) Insight into nanoparticle cellular uptake and intracellular targeting. $J$ Control Release 190: 485-499.

Zhang C, Tang N, Liu X, Liang W, Xu W, and Torchilin VP (2006) siRNA-containing liposomes modified with polyarginine effectively silence the targeted gene. $J$ Control Release 112:229-239.

Zhang Q, Wang J, Zhang H, Liu D, Ming L, Liu L, Dong Y, Jian B, and Cai D (2018) The anticancer efficacy of paclitaxel liposomes modified with low-toxicity hydrophobic cell-penetrating peptides in breast cancer: an: in vitro and in vivo evaluation. RSC Advances 8:24084-24093.

Zheng C, Ma C, Bai E, Yang K, and Xu R (2015) Transferrin and cell-penetrating peptide dual-functioned liposome for targeted drug delivery to glioma. Int J Clin Exp Med 8:1658-1668.

Zhou Y, Peng Z, Seven ES, and Leblanc RM (2018) Crossing the blood-brain barrier with nanoparticles. J Control Release 270:290-303.

Zou LL, Huang L, Hayes RL, Black C, Qiu YH, Perez-Polo JR, Le W, Clifton GL, and Yang K (1999) Liposome-mediated NGF gene transfection following neuronal injury: potential therapeutic applications. Gene Ther 6:994-1005.

Zylberberg C, Gaskill K, Pasley S, and Matosevic S (2017) Engineering liposomal nanoparticles for targeted gene therapy. Gene Ther 24:441-452.

Address correspondence to: Jagdish Singh, Department of Pharmaceutical Sciences, School of Pharmacy, College of Health Professions, North Dakota State University, Fargo, ND 58105. E-mail: jagdish.singh@ndsu.edu 